1173

\section{AN INVESTIGATION ON THE STATUS OF TERM, PREMATURE AND LOW BIRTH WEIGHT AND ITS ASSOCIATION WITH SOME MATERNAL FACTORS}

\author{
P. Taheri \\ Paediatric Nursing Dept, Isfahan University of \\ Medical Sciences, Isfahan, Iran
}

Background and aims: Low birth weight is one of most important new born mortality factors. The cause of this mortality is either being premature or low birth weight of new borns and congenital diseases.

Methods: The population studied comprised 380 term, premature and low birth weight in NICU wards. The data were collected by questionnaires composed of three sections. The first section contained questions on mothers' personal demographic characteristics and the second included mothers' social characteristics. The third was composed of questions on new born's.

Results: Term, premature and low weight new born's status was questioned from the mother, investigated in the infants' files and then was recorded. The findings showed that $57.1 \%$ of the infants were boys and $46.6 \%$ girls. Mothers mean age based on new borns' birth weight through spearman correlation test shows a non significant association between birth weight and mothers' age, ( $r=\% 2 \mathrm{P}=0.698$ ). (Analyzing)Mothers' mean age pre pregnancy based on infants' weight status through speasrman correlation test shows a non significant association between infants' birth weight and pre pregnancy mothres' weight $(p=0.883)$. This association for the post pregnancy mothers' weight is the same $(p=0.117, r=0.08)$.

Conclusions: Although, the findings of the research showed no significant association between birth weight with parents' education, food consumption, medication, height, age and gender, there was a significant association between birth weight with infants' APGAR.

Keywords: Mothers' personal and social economic social economic factors, with term, premature and low birth weight ).

\section{RISK FACTORS FOR RETINOPATHY OF PREMATURITY AMONG PRETERM IN ONE OF THE LARGEST SAUDI'S WESTERN REGION NEONATAL INTENSIVE CARE UNITS}

\author{
M. Abdulrahim ${ }^{1}$, M. Montasser ${ }^{1}$, \\ M. Abou Al-Seoud ${ }^{1}$, D. EL Metwally ${ }^{1,2}$ \\ ${ }^{1}$ Neonatology, King Fahd Armed Forces Hospital, \\ Jeddah, Saudi Arabia, ${ }^{2}$ Pediatrics, Faculty of \\ Medicine, Suez Canal University, Ismailia, Egypt
}

Background and aims: To detect 1- the prevalence of severe retinopathy of prematurity (> stage 2 International classification of ROP) among infants $\leq 32$ weeks of gestation. 2- The risk factors that are associated with severe ROP.

Methods: Aretrospective study on infants $\leq 32$ weeks born between January 2007 and October 2009 at the King Fahd Armed Forces Hospital of Jeddah in Saudi Arabia. Ophthalmologic examinations as per AAP recommendation was carried till resolution.

Results: There were 218 infants $\leq 32 \mathrm{wks}$, the rate of $R O P>2$ was $14.3 \%$ of whom: $48.3 \%$ had plus disease, $55.1 \%$ had laser and $4.4 \%$ expired prior to discharge. Birth-weight(BW) and gestationalage(GA): $1444 \pm 385$ vs $872 \pm 206$ and $32.2 \pm 2$ vs $26.7 \pm 2$ among $R O P>2$ and No-ROP respectively, $p<$ 0.0001 None of the exclusively breast fed had (12\%) had ROP $>2$. Post-natal growth differences between ROP $>2$ vs No-ROP was clinically and statistically insignificant. $86 \%$ of $\mathrm{ROP}>2$ had mechanical ventilation, $p<.001$. Among the 12 infants requiring HFOV, $83.3 \%$ had ROP>2, $p<.0001 .76 \%$ of ROP $>2$ were on CPAP. BPD occurred among 32 infants; $48.3 \%$ had ROP $>$ II, $p<.0001$. Eight infants required respiratory support at 36 wks PMA, 5 $(63 \%)$ had ROP>II, $p<.002$. Positive culture sepsis occurred among $37.9 \%$ of ROP $>2.52 \%$ of ROP $>2$ received blood transfusion, $p<.02$. There was no statistical significant relation between IVH $\geq I I I, P V L$, Hydrocephalus, NEC, PDA, LOS and ROP $>2$.

Conclusion: Exclusive breast feeding seems to be protective of severe ROP. Post-natal growth was not a risk factor however, GA, BW and ventilation were the major determinant of severe ROP. 